\title{
Research Article \\ Stability of a Mixed Type Functional Equation on Multi-Banach Spaces: A Fixed Point Approach
}

\author{
Liguang Wang, Bo Liu, and Ran Bai \\ School of Mathematical Sciences, Qufu Normal University, Qufu 273165, China \\ Correspondence should be addressed to Liguang Wang, wangliguang0510@163.com
}

Received 11 December 2009; Accepted 29 March 2010

Academic Editor: Marlène Frigon

Copyright @ 2010 Liguang Wang et al. This is an open access article distributed under the Creative Commons Attribution License, which permits unrestricted use, distribution, and reproduction in any medium, provided the original work is properly cited.

Using fixed point methods, we prove the Hyers-Ulam-Rassias stability of a mixed type functional equation on multi-Banach spaces.

\section{Introduction and Preliminaries}

The stability problem of functional equations originated from a question of Ulam [1] concerning the stability of group homomorphisms. Hyers [2] gave a first affirmative partial answer to the question of Ulam for Banach spaces. Hyers's theorem was generalized by Aoki [3] for additive mappings and by Rassias [4] for linear mappings by considering an unbounded Cauchy difference. The paper of Rassias has provided a lot of influence in the development of what we call generalized Hyers-Ulam-Rassias stability of functional equations. In 1990, Rassias [5] asked whether such a theorem can also be proved for $p \geq 1$. In 1991, Gajda [6] gave an affirmative solution to this question when $p>1$, but it was proved by Gajda [6] and Rassias and Šemrl [7] that one cannot prove an analogous theorem when $p=1$. In 1994, a generalization was obtained by Gavruta [8], who replaced the bound $\varepsilon\left(\|x\|^{p}+\|y\|^{p}\right)$ by a general control function $\phi(x, y)$. Beginning around 1980, the stability problems of several functional equations and approximate homomorphisms have been extensively investigated by a number of authors, and there are many interesting results concerning this problem. Some of the open problems in this field were solved in the papers mentioned [9-15].

The notion of multi-normed space was introduced by Dales and Polyakov (see in [1619]). This concept is somewhat similar to operator sequence space and has some connections with operator spaces and Banach lattices. Motivations for the study of multi-normed spaces and many examples were given in [16]. Let $(E,\|\cdot\|)$ be a complex linear space, and let $K \in \mathbb{N}$, we denote by $E^{k}$ the linear space $E \oplus \cdots \oplus E$ consisting of $k$-tuples $\left(x_{1}, \ldots, x_{k}\right)$, where $x_{1}, \ldots, x_{k} \in E$. The linear operations on $E^{k}$ are defined coordinate-wise. When we write 
$\left(0, \ldots, 0, x_{i}, 0, \ldots, 0\right)$ for an element in $E^{k}$, we understand that $x_{i}$ appears in the $i$ th coordinate. The zero elements of either $E$ or $E^{k}$ are both denoted by 0 when there is no confusion. We denote by $\mathbb{N}_{k}$ the set $\{1,2, \ldots, k\}$ and by $\mathbb{B}_{k}$ the group of permutations on $\mathbb{N}_{k}$.

Definition 1.1. A multi-norm on $\left\{E^{n}, n \in \mathbb{N}\right\}$ is a sequence

$$
\left(\|\cdot\|_{n}\right)=\left(\|\cdot\|_{n}: n \in \mathbb{N}\right)
$$

such that $\|\cdot\|_{n}$ is a norm on $E^{n}$ for each $n \in \mathbb{N}$, such that $\|x\|_{1}=\|x\|$ for each $x \in E$, and such that for each $n \in \mathbb{N}(n \geq 2)$, the following axioms are satisfied:

$$
\begin{aligned}
& \left(A_{1}\right) \|\left(x_{\sigma(1)}, \ldots, x_{\sigma(n)}\left\|_{n}=\right\|\left(x_{1}, \ldots, x_{n}\right) \|_{n}\left(\forall \sigma \in B_{n}, x_{1}, \ldots, x_{n} \in E\right)\right. \\
& \left(A_{2}\right)\left\|\left(\alpha_{1} x_{1}, \ldots, \alpha_{n} x_{n}\right)\right\|_{n} \leq\left(\max _{i \in \mathbb{N}_{n}}\left|\alpha_{i}\right|\right)\left\|\left(x_{1}, \ldots, x_{n}\right)\right\|_{n}\left(x_{i} \in E, \alpha_{i} \in \mathbb{C}, i=1, \ldots, n\right) ; \\
& \left(A_{3}\right)\left\|\left(x_{1}, \ldots, x_{n-1}, 0\right)\right\|_{n}=\left\|\left(x_{1}, \ldots, x_{n-1}\right)\right\|_{n-1}\left(x_{1}, \ldots, x_{n-1} \in E\right) \\
& \left(A_{4}\right)\left\|\left(x_{1}, \ldots, x_{n-1}, x_{n-1}\right)\right\|_{n}=\left\|\left(x_{1}, \ldots, x_{n-1}\right)\right\|_{n-1}\left(x_{1}, \ldots, x_{n-1} \in E\right) .
\end{aligned}
$$

In this case, we say that $\left(\left(E^{n},\|\cdot\|_{n}\right): n \in \mathbb{N}\right)$ is a multi-normed space.

Suppose that $\left(\left(E^{n},\|\cdot\|_{n}\right): n \in \mathbb{N}\right)$ is a multi-normed space and $k \in \mathbb{N}$. It is easy to show that

(a) $\|(x, \ldots, x)\|_{k}=\|x\|(x \in E)$;

(b) $\max _{i \in \mathbb{N}_{k}}\left\|x_{i}\right\| \leq\left\|\left(x_{1}, \ldots, x_{k}\right)\right\|_{k} \leq \sum_{i=1}^{k}\left\|x_{i}\right\| \leq k \max _{i \in \mathbb{N}_{k}}\left\|x_{i}\right\|\left(x_{1}, \ldots, x_{k} \in E\right)$.

It follows from (b) that if $(E,\|\cdot\|)$ is a Banach space, then $\left(E^{k},\|\cdot\|_{k}\right)$ is a Banach space for each $k \in \mathbb{N}$; in this case $\left(\left(E^{k},\|\cdot\|_{k}\right): k \in \mathbb{N}\right)$ is said to be a multi-Banach space.

In the following, we first recall some fundamental result in fixed-point theory.

Let $X$ be a set. A function $d: X \times X \rightarrow[0, \infty]$ is called a generalized metric on $X$ if $d$ satisfies

(1) $d(x, y)=0$ if and only if $x=y$;

(2) $d(x, y)=d(y, x)$ for all $x, y \in X$;

(3) $d(x, z) \leq d(x, y)+d(y, z)$ for all $x, y, z \in X$.

We recall the following theorem of Diaz and Margolis [20].

Theorem 1.2 (see [20]). let $(X, d)$ be a complete generalized metric space and let $J: X \rightarrow X$ be a strictly contractive mapping with Lipschitz constant $0<L<1$. Then for each given element $x \in X$, either

$$
d\left(J^{n} x, J^{n+1} x\right)=\infty
$$

for all nonnegative integers $n$ or there exists a nonnegative integer $n_{0}$ such that

(1) $d\left(J^{n} x, J^{n+1} x\right)<\infty$ for all $n \geq n_{0}$;

(2) the sequence $\left\{J^{n} x\right\}$ converges to a fixed point $y^{*}$ of $J$;

(3) $y^{*}$ is the unique fixed point of $J$ in the set $Y=\left\{y \in X: d\left(J^{n_{0}} x, y\right)<\infty\right\}$;

(4) $d\left(y, y^{*}\right) \leq 1 /(1-L) d(y, J y)$ for all $y \in Y$. 
Baker [21] was the first author who applied the fixed-point method in the study of Hyers-Ulam stability (see also [22]). In 2003, Cadariu and Radu applied the fixed-point method to the investigation of the Jensen functional equation (see [23, 24]). By using fixed point methods, the stability problems of several functional equations have been extensively investigated by a number of authors (see [25-27]).

In this paper, we will show the Hyers-Ulam-Rassias stability of a mixed type functional equation on multi-Banach spaces using fixed-point methods.

\section{A Mixed Type Functional Equation}

In this section, we investigate the stability of the following functional equation in multiBanach spaces:

$$
\begin{aligned}
f(x+2 y)+f(x-2 y)= & 4 f(x+y)+4 f(x-y)-6 f(x)+f(4 y)-4 f(3 y) \\
& +6 f(2 y)-4 f(y) .
\end{aligned}
$$

Let

$$
\begin{aligned}
D f(x, y)= & f(x+2 y)+f(x-2 y)-4 f(x+y)-4 f(x-y)+6 f(x)-f(4 y) \\
& +4 f(3 y)-6 f(2 y)+4 f(y) .
\end{aligned}
$$

First we give some lemma needed later.

Lemma 2.1 (see [28] Lemma 6.1). If an even function $f: X \rightarrow Y$ satisfies(2.1), then $f$ is quarticquadratic function.

Lemma 2.2 (see [28] Lemma 6.2). If an odd function $f: X \rightarrow Y$ satisfies (2.1), then $f$ is cubicadditive function.

Theorem 2.3. Let E be a linear space and let $\left(\left(F^{n},\|\cdot\|_{n}\right): n \in \mathbb{N}\right)$ be a multi-Banach space. Let $k \in \mathbb{N}$ and let $f: E \rightarrow F$ be an even mapping with $f(0)=0$ for which there exists a positive real number $\epsilon$ such that

$$
\sup _{k \in \mathbb{N}}\left\|\left(D f\left(x_{1}, y_{1}\right), \ldots, D f\left(x_{k}, y_{k}\right)\right)\right\|_{k} \leq \epsilon
$$

for all $x_{1}, \ldots, x_{k}, y_{1}, \ldots, y_{k} \in E(k \in \mathbb{N})$. Then there exists a unique quadratic mapping $Q_{1}: E \rightarrow F$ satisfying (2.1) and

$$
\sup _{k \in \mathbb{N}}\left\|\left(f\left(2 x_{1}\right)-16 f\left(x_{1}\right)-Q\left(x_{1}\right), \ldots, f\left(2 x_{k}\right)-16 f\left(x_{k}\right)-Q\left(x_{k}\right)\right)\right\|_{k} \leq 3 \epsilon
$$

for all $x_{1}, \ldots, x_{k} \in E$. 
Proof. Putting $x_{1}=\cdots=x_{k}=0$ in (2.3), we have

$$
\begin{aligned}
\sup _{k \in \mathbb{N}} \|( & f\left(4 y_{1}\right)-4 f\left(3 y_{1}\right)+4 f\left(2 y_{1}\right)+4 f\left(y_{1}\right), \ldots, f\left(4 y_{k}\right)-4 f\left(3 y_{k}\right) \\
+ & \left.4 f\left(2 y_{k}\right)+4 f\left(y_{k}\right)\right) \|_{k} \leq \epsilon .
\end{aligned}
$$

Replacing $x_{i}$ with $y_{i}$ in (2.3), we get

$$
\begin{aligned}
& \sup _{k \in \mathbb{N}} \|( f\left(4 y_{1}\right)+5 f\left(3 y_{1}\right)-10 f\left(2 y_{1}\right)+11 f\left(y_{1}\right), \ldots,-f\left(4 y_{k}\right)+5 f\left(3 y_{k}\right) \\
&\left.-10 f\left(2 y_{k}\right)+11 f\left(y_{k}\right)\right) \|_{k} \leq \epsilon .
\end{aligned}
$$

By (2.5) and (2.6), we have

$$
\sup _{k \in \mathbb{N}}\left\|\left(f\left(4 x_{1}\right)-20 f\left(2 x_{1}\right)+64 f\left(x_{1}\right), \ldots, f\left(4 x_{k}\right)-20 f\left(2 x_{k}\right)+64 f\left(x_{k}\right)\right)\right\|_{k} \leq 9 \epsilon .
$$

Let $J(x)=f(2 x)-16 f(x)$ for all $x \in X$. Then we have

$$
\sup _{k \in \mathbb{N}}\left\|\left(J\left(2 x_{1}\right)-4 J\left(x_{1}\right), \ldots, J\left(2 x_{k}\right)-4 J\left(x_{k}\right)\right)\right\|_{k} \leq 9 \epsilon .
$$

Set $X=\{g: E \rightarrow F: g(0)=0\}$ and define a metric $d$ on $X$ by

$$
\begin{gathered}
d(g, h)=\inf \left\{c>0: \sup _{k \in \mathbb{N}}\left\|g\left(x_{1}\right)-h\left(x_{1}\right), \ldots, g\left(x_{k}\right)-h\left(x_{k}\right)\right\|_{k} \leq c:\right. \\
\left.x_{1}, \ldots, x_{k} \in \mathbb{N}, k \in \mathbb{N}\right\} .
\end{gathered}
$$

Define a map $\Lambda: X \rightarrow X$ by $\Lambda(g)(x)=(g(2 x)) / 4$. Let $g, h \in X$ and let $c \in[0, \infty]$ be an arbitrary constant with $d(g, h) \leq c$. From the definition of $d$, we have

$$
\sup _{k \in \mathbb{N}}\left\|g\left(x_{1}\right)-h\left(x_{1}\right), \ldots, g\left(x_{k}\right)-h\left(x_{k}\right)\right\|_{k} \leq c
$$

for $x_{1}, \ldots, x_{k} \in \mathbb{N}, k \in \mathbb{N}$. Then

$$
\begin{aligned}
\sup _{k \in \mathbb{N}} \|( & (\Lambda g)\left(x_{1}\right)-(\Lambda h)\left(x_{1}\right), \ldots,(\Lambda g)\left(x_{k}\right)-(\Lambda h)\left(x_{k}\right) \|_{k} \\
& \leq \frac{1}{4} \sup _{k \in \mathbb{N}}\left\|g\left(2 x_{1}\right)-h\left(2 x_{1}\right), \ldots, g\left(2 x_{k}\right)-h\left(2 x_{k}\right)\right\|_{k} \leq \frac{c}{4}
\end{aligned}
$$


for $x_{1}, \ldots, x_{k} \in \mathbb{N}, k \in \mathbb{N}$. So

$$
d(\Lambda g, \Lambda h) \leq \frac{1}{4} d(g, h)
$$

Then $\Lambda$ is a strictly contractive mapping. It follows from (2.8) that

$$
\begin{aligned}
\sup _{k \in \mathbb{N}} \|( & \Lambda J)\left(x_{1}\right)-J\left(x_{1}\right), \ldots,(\Lambda J)\left(x_{k}\right)-J\left(x_{k}\right) \|_{k} \\
& \leq \frac{1}{4} \sup _{k \in \mathbb{N}}\left\|J\left(2 x_{1}\right)-4 J\left(2 x_{1}\right), \ldots, J\left(2 x_{k}\right)-4 J\left(2 x_{k}\right)\right\|_{k} \leq \frac{9 \epsilon}{4}
\end{aligned}
$$

for $x_{1}, \ldots, x_{k} \in \mathbb{N}, k \in \mathbb{N}$. Then $d(\Lambda J, J) \leq 9 \epsilon / 4$. According to Theorem 1.2, the sequence $\left\{\Lambda^{n} J\right\}$ converges to a unique fixed point $Q_{1}$ of $\Lambda$ in $X$, that is,

$$
\begin{gathered}
Q_{1}(x)=\lim _{n \rightarrow \infty}\left(\Lambda^{n} J\right)(x)=\lim _{n \rightarrow \infty} \frac{1}{4^{n}} J\left(2^{n} x\right), \\
d\left(J, Q_{1}\right) \leq \frac{4}{3} d(\Lambda J, J)=3 \epsilon .
\end{gathered}
$$

Also we have $(Q(2 x)) / 4=Q(x)$ for all $x \in X$, that is, $Q(2 x)=4 Q(x)$ for all $x \in X$. Also we have

$$
\begin{aligned}
D Q_{1}(x, y) & =\lim _{n \rightarrow \infty} \frac{1}{4^{n}}\left\|D J\left(2^{n} x, 2^{n} y\right)\right\|=\lim _{n \rightarrow \infty} \frac{1}{4^{n}}\left\|D f\left(2^{n+1} x, 2^{n+1} y\right)-16 D f\left(2^{n} x, 2^{n} y\right)\right\| \\
& \leq \lim _{n \rightarrow \infty} \frac{17 \epsilon}{4^{n}}=0
\end{aligned}
$$

and $Q_{1}$ satisfies (2.1). Since $Q_{1}$ is also even and $Q_{1}(0)=0$, we have that $Q(2 x)-16 Q(x)=$ $-12 Q(x)$ is quadratic by Lemma 2.1. Then $Q$ is quadratic.

Theorem 2.4. Let E be a linear space and let $\left(\left(F^{n},\|\cdot\|_{n}\right): n \in \mathbb{N}\right)$ be a multi-Banach space. Let $k \in \mathbb{N}$ and let $f: E \rightarrow F$ be an even mapping with $f(0)=0$ for which there exists a positive real number $\epsilon$ such that (2.3) holds for all $x_{1}, \ldots, x_{k}, y_{1}, \ldots, y_{k} \in E(k \in \mathbb{N})$. Then there exists a unique quartic mapping $Q_{2}: E \rightarrow F$ satisfying (2.1) and

$$
\sup _{k \in \mathbb{N}}\left\|\left(f\left(2 x_{1}\right)-4 f\left(x_{1}\right)-Q_{2}\left(x_{1}\right), \ldots, f\left(2 x_{k}\right)-4 f\left(x_{k}\right)-Q_{2}\left(x_{k}\right)\right)\right\|_{k} \leq \frac{3}{5} \epsilon
$$

for all $x_{1}, \ldots, x_{k} \in E$.

Proof. The proof is similar to that of Theorem 2.3.

Theorem 2.5. Let $E$ be a linear space and let $\left(\left(F^{n},\|\cdot\|_{n}\right): n \in \mathbb{N}\right)$ be a multi-Banach space. Let $k \in \mathbb{N}$ and let $f: E \rightarrow F$ be an even mapping with $f(0)=0$ for which there exists a positive real number $\epsilon$ 
such that (2.3) holds for all $x_{1}, \ldots, x_{k}, y_{1}, \ldots, y_{k} \in E(k \in \mathbb{N})$. Then there exist a unique quadratic mapping $Q_{1}: E \rightarrow F$ and a unique quadratic mapping $Q_{2}: E \rightarrow F$ such that

$$
\sup _{k \in \mathbb{N}}\left\|\left(f\left(x_{1}\right)-Q_{1}\left(x_{1}\right)-Q_{2}\left(x_{1}\right), \ldots, f\left(x_{k}\right)-Q_{1}\left(x_{k}\right)-Q_{2}\left(x_{k}\right)\right)\right\|_{k} \leq \frac{3 \epsilon}{10}
$$

for all $x_{1}, \ldots, x_{k} \in E$.

Proof. By Theorems 2.3 and 2.4, there exist a quadratic mapping $Q_{1}^{0}: E \rightarrow F$ and a unique quartic mapping $Q_{2}^{0}: E \rightarrow f$ such that

$$
\begin{gathered}
\sup _{k \in \mathbb{N}}\left\|\left(f\left(2 x_{1}\right)-16 f\left(x_{1}\right)-Q_{1}^{0}\left(x_{1}\right), \ldots, f\left(2 x_{k}\right)-16 f\left(x_{k}\right)-Q_{1}^{0}\left(x_{k}\right)\right)\right\|_{k} \leq 3 \epsilon \\
\sup _{k \in \mathbb{N}}\left\|\left(f\left(2 x_{1}\right)-4 f\left(x_{1}\right)-Q_{2}^{0}\left(x_{1}\right), \ldots, f\left(2 x_{k}\right)-4 f\left(x_{k}\right)-Q_{2}^{0}\left(x_{k}\right)\right)\right\|_{k} \leq \frac{3}{5} \epsilon
\end{gathered}
$$

for all $x_{1}, \ldots, x_{k} \in E$. By (2.18), we have

$$
\sup _{k \in \mathbb{N}}\left\|\left(12 f\left(x_{1}\right)+Q_{1}^{0}\left(x_{1}\right)-Q_{2}^{0}\left(x_{1}\right), \ldots, 12 f\left(x_{k}\right)+Q_{1}^{0}\left(x_{k}\right)-Q_{2}^{0}\left(x_{k}\right)\right)\right\|_{k} \leq \frac{18}{5} \epsilon .
$$

Let $Q_{1}(x)=-(1 / 12) Q_{1}^{0}(x)$ and $Q_{2}(x)=(1 / 12) Q_{2}^{0}(x)$ for all $x \in E$. Then we have (2.17). The uniqueness of $Q_{1}$ and $Q_{2}$ is easy to show.

Theorem 2.6. Let $E$ be a linear space and let $\left(\left(F^{n},\|\cdot\|_{n}\right): n \in \mathbb{N}\right)$ be a multi-Banach space. Let $k \in \mathbb{N}$ and let $f: E \rightarrow F$ be an odd mapping for which there exists a positive real number $\epsilon$ such that (2.3) holds for all $x_{1}, \ldots, x_{k}, y_{1}, \ldots, y_{k} \in E(k \in \mathbb{N})$. Then there exists a unique additive mapping $A: E \rightarrow F$ and a unique cubic mapping $C: E \rightarrow F$ satisfying (2.1) and

$$
\begin{gathered}
\sup _{k \in \mathbb{N}}\left\|\left(f\left(2 x_{1}\right)-8 f\left(x_{1}\right)-A\left(x_{1}\right), \ldots, f\left(2 x_{k}\right)-8 f\left(x_{k}\right)-A\left(x_{k}\right)\right)\right\|_{k} \leq 9 \epsilon, \\
\sup _{k \in \mathbb{N}}\left\|\left(f\left(2 x_{1}\right)-2 f\left(x_{1}\right)-C\left(x_{1}\right), \ldots, f\left(2 x_{k}\right)-f\left(x_{k}\right)-C\left(x_{k}\right)\right)\right\|_{k} \leq \frac{9}{7} \epsilon
\end{gathered}
$$

for all $x_{1}, \ldots, x_{k} \in E$.

Proof. The proof is similar to that of Theorems 2.3 and 2.4 .

Theorem 2.7. Let $E$ be a linear space and let $\left(\left(F^{n},\|\cdot\|_{n}\right): n \in \mathbb{N}\right)$ be a multi-Banach space. Let $k \in \mathbb{N}$ and let $f: E \rightarrow F$ be an odd mapping for which there exists a positive real number $\epsilon$ such that (2.3) holds for all $x_{1}, \ldots, x_{k}, y_{1}, \ldots, y_{k} \in E(k \in \mathbb{N})$. Then there exists a unique additive mapping $A: E \rightarrow F$ and a unique cubic mapping $C: E \rightarrow F$ satisfying (2.1) and

$$
\sup _{k \in \mathbb{N}}\left\|\left(f\left(x_{1}\right)-A\left(x_{1}\right)-C\left(x_{1}\right), \ldots, f\left(x_{k}\right)-A\left(x_{k}\right)-C\left(x_{k}\right)\right)\right\|_{k} \leq \frac{12}{7} \epsilon
$$

for all $x_{1}, \ldots, x_{k} \in E$. 
Proof. By Theorem 2.6, there is an additive mapping $A_{0}: E \rightarrow F$ and a cubic mapping $C_{0}$ : $E \rightarrow F$ such that

$$
\begin{aligned}
& \sup _{k \in \mathbb{N}}\left\|\left(f\left(2 x_{1}\right)-8 f\left(x_{1}\right)-A_{0}\left(x_{1}\right), \ldots, f\left(2 x_{k}\right)-8 f\left(x_{k}\right)-A_{0}\left(x_{k}\right)\right)\right\|_{k} \leq 9 \epsilon, \\
& \sup _{k \in \mathbb{N}}\left\|\left(f\left(2 x_{1}\right)-2 f\left(x_{1}\right)-C_{0}\left(x_{1}\right), \ldots, f\left(2 x_{k}\right)-2 f\left(x_{k}\right)-C_{0}\left(x_{k}\right)\right)\right\|_{k} \leq \frac{9}{7} \epsilon .
\end{aligned}
$$

Thus

$$
\sup _{k \in \mathbb{N}}\left\|\left(6 f\left(x_{1}\right)+A_{0}\left(x_{1}\right)-C_{0}\left(x_{1}\right), \ldots, 6 f\left(x_{k}\right)+A_{0}\left(x_{k}\right)-C_{0}\left(x_{k}\right)\right)\right\|_{k} \leq \frac{72}{7} \epsilon
$$

for all $x_{1}, \ldots, x_{k} \in E$. Let $A=-A_{0} / 6$ and $C=C_{0} / 6$. The rest is similar to that of the proof of Theorem 2.5.

Theorem 2.8. Let E be a linear space and let $\left(\left(F^{n},\|\cdot\|_{n}\right): n \in \mathbb{N}\right)$ be a multi-Banach space. Let $k \in \mathbb{N}$ and let $f: E \rightarrow F$ be an odd mapping satisfying $f(0)=0$ and there exists a positive real number $\epsilon$ such that (2.3) holds for all $x_{1}, \ldots, x_{k}, y_{1}, \ldots, y_{k} \in E(k \in \mathbb{N})$. Then there exist a unique additive mapping $A: E \rightarrow F$, a unique cubic mapping $C: E \rightarrow F$, a unique quadratic mapping $Q_{1}: E \rightarrow F$, and a unique quadratic mapping $Q_{2}: E \rightarrow F$ such that

$$
\begin{gathered}
\sup _{k \in \mathbb{N}} \|\left(f\left(x_{1}\right)-A\left(x_{1}\right)-Q\left(x_{1}\right)-C\left(x_{1}\right)-Q_{2}\left(x_{1}\right), \ldots, f\left(x_{k}\right)-A\left(x_{k}\right)-Q_{1}\left(x_{k}\right)\right. \\
\left.-C\left(x_{k}-Q_{2}\left(x_{k}\right)\right)\right) \|_{k} \leq \frac{141}{70} \epsilon
\end{gathered}
$$

for all $x_{1}, \ldots, x_{k} \in E$.

Proof. Let $f_{e}(x)=1 / 2(f(x)+f(-x))$ for all $x \in E$. Then $f_{e}(0)=0$ and $f_{e}(-x)=f_{e}(x)$ and

$$
\sup _{k}\left\|D f_{e}\left(x_{1}, y_{1}\right), \ldots, D f_{e}\left(x_{k}, y_{k}\right)\right\|_{k} \leq \epsilon
$$

for all $x_{1}, \ldots, x_{k}, y_{1}, \ldots, y_{k} \in E$. By Theorem 2.5, there are a unique quadratic mapping $Q_{1}$ : $E \rightarrow F$ and a unique quartic mapping $Q_{2}: E \rightarrow F$ satisfying

$$
\sup _{k \in \mathbb{N}}\left\|\left(f_{e}\left(x_{1}\right)-Q_{1}\left(x_{1}\right)-Q_{2}\left(x_{1}\right), \ldots, f_{e}\left(x_{k}\right)-Q_{1}\left(x_{k}\right)-Q_{2}\left(x_{k}\right)\right)\right\|_{k} \leq \frac{3 \epsilon}{10} .
$$

Let $f_{o}(x)=1 / 2(f(x)-f(-x))$ for all $x \in E$. Then $f_{o}$ is an odd mapping satisfying

$$
\sup _{k}\left\|D f_{o}\left(x_{1}, y_{1}\right), \ldots, D f_{o}\left(x_{k}, y_{k}\right)\right\|_{k} \leq \epsilon
$$


for all $x_{1}, \ldots, x_{k}, y_{1}, \ldots, y_{k} \in E$. By Theorem 2.7, there are a unique additive mapping $A: E \rightarrow$ $F$ and a unique quartic mapping $C: E \rightarrow F$ satisfying

$$
\sup _{k \in \mathbb{N}}\left\|\left(f_{o}\left(x_{1}\right)-A\left(x_{1}\right)-C\left(x_{1}\right), \ldots, f\left(x_{k}\right)-A\left(x_{k}\right)-C\left(x_{k}\right)\right)\right\|_{k} \leq \frac{12}{7} \epsilon .
$$

By (2.26) and (2.28), we have (2.24).This completes the proof.

\section{Acknowledgments}

This work was supported in part by the Scientific Research Project of the Department of Education of Shandong Province (no. J08LI15). The authors are grateful to the referees for their valuable suggestions.

\section{References}

[1] S. M. Ulam, A Collection of Mathematical Problems, Interscience Tracts in Pure and Applied Mathematics, no. 8, Interscience, New York, NY, USA, 1960.

[2] D. H. Hyers, "On the stability of the linear functional equation," Proceedings of the National Academy of Sciences of the United States of America, vol. 27, pp. 222-224, 1941.

[3] T. Aoki, "On the stability of the linear transformation in Banach spaces," Journal of the Mathematical Society of Japan, vol. 2, pp. 64-66, 1950.

[4] Th. M. Rassias, "On the stability of the linear mapping in Banach spaces," Proceedings of the American Mathematical Society, vol. 72, no. 2, pp. 297-300, 1978.

[5] Th. M. Rassias, Ed., Functional Equations, Inequalities and Applications, Kluwer Academic Publishers, Dordrecht, The Netherlands, 2003.

[6] Z. Gajda, "On stability of additive mappings," International Journal of Mathematics and Mathematical Sciences, vol. 14, no. 3, pp. 431-434, 1991.

[7] Th. M. Rassias and P. Šemrl, "On the behavior of mappings which do not satisfy Hyers-Ulam stability," Proceedings of the American Mathematical Society, vol. 114, no. 4, pp. 989-993, 1992.

[8] P. Gavruta, "A generalization of the Hyers-Ulam-Rassias stability of approximately additive mappings," Journal of Mathematical Analysis and Applications, vol. 184, no. 3, pp. 431-436, 1994.

[9] P. Gavruta, "An answer to a question of Th. M. Rassias and J. Tabor on mixed stability of mappings," Buletinul ŞtiinŞific al Universităţii "Politehnica" din Timişoara. Seria Matematică-Fizică, vol. 42(56), no. 1, pp. 1-6, 1997.

[10] P. Gavruta, "On the Hyers-Ulam-Rassias stability of mappings," in Recent Progress in Inequalities, vol. 430 of Mathematics and Its Applications, pp. 465-469, Kluwer Academic Publishers, Dordrecht, The Netherlands, 1998.

[11] P. Gavruta, "An answer to a question of John M. Rassias concerning the stability of Cauchy equation," in Advances in Equations and Inequalities, Hadronic Mathematics Series, pp. 67-71, 1999.

[12] P. Gavruta, "On a problem of G. Isac and Th. M. Rassias concerning the stability of mappings," Journal of Mathematical Analysis and Applications, vol. 261, no. 2, pp. 543-553, 2001.

[13] P. Gavruta, "On the Hyers-Ulam-Rassias stability of the quadratic mappings," Nonlinear Functional Analysis and Applications, vol. 9, no. 3, pp. 415-428, 2004.

[14] P. Gavruta, M. Hossu, D. Popescu, and C. Căprău, “On the stability of mappings and an answer to a problem of Th. M. Rassias," Annales Mathématiques Blaise Pascal, vol. 2, no. 2, pp. 55-60, 1995.

[15] L. Gavruta and P. Gavruta, "On a problem of John M. Rassias concerning the stability in Ulam sense of Euler-Lagrange equation," in Functional Equations, Difference Inequalities and Ulam Stability Notions, pp. 47-53, Nova Sciences, 2010.

[16] H. G. Dales and M. E. Polyakov, "Multi-normed spaces and multi-Banach algebras," preprint.

[17] H. G. Dales and M. S. Moslehian, "Stability of mappings on multi-normed spaces," Glasgow Mathematical Journal, vol. 49, no. 2, pp. 321-332, 2007. 
[18] M. S. Moslehian, K. Nikodem, and D. Popa, "Asymptotic aspect of the quadratic functional equation in multi-normed spaces," Journal of Mathematical Analysis and Applications, vol. 355, no. 2, pp. 717-724, 2009.

[19] M. S. Moslehian, "Superstability of higher derivations in multi-Banach algebras," Tamsui Oxford Journal of Mathematical Sciences, vol. 24, no. 4, pp. 417-427, 2008.

[20] J. B. Diaz and B. Margolis, "A fixed point theorem of the alternative, for contractions on a generalized complete metric space," Bulletin of the American Mathematical Society, vol. 74, pp. 305-309, 1968.

[21] J. A. Baker, "The stability of certain functional equations," Proceedings of the American Mathematical Society, vol. 112, no. 3, pp. 729-732, 1991.

[22] R. P. Agarwal, B. Xu, and W. Zhang, "Stability of functional equations in single variable," Journal of Mathematical Analysis and Applications, vol. 288, no. 2, pp. 852-869, 2003.

[23] L. Cadariu and V. Radu, "Fixed points and the stability of Jensen's functional equation," Journal of Inequalities in Pure and Applied Mathematics, vol. 4, no. 1, article 4, 7 pages, 2003.

[24] L. Cadariu and V. Radu, "On the stability of the Cauchy functional equation: a fixed point approach," in Iteration Theory (ECIT '02), vol. 346 of Die Grazer Mathematischen Berichte, pp. 43-52, Karl-FranzensUniversitaet Graz, Graz, Austria, 2004.

[25] S.-M. Jung and J. M. Rassias, "A fixed point approach to the stability of a functional equation of the spiral of Theodorus," Fixed Point Theory and Applications, vol. 2008, Article ID 945010, 7 pages, 2008.

[26] C. Park and J. M. Rassias, "Stability of the Jensen-type functional equation in $C^{*}$-algebras: a fixed point approach," Abstract and Applied Analysis, vol. 2009, Article ID 360432, 17 pages, 2009.

[27] C. Park and J. S. An, "Stability of the Cauchy-Jensen functional equation in $C^{*}$-algebras: a fixed point approach," Fixed Point Theory and Applications, vol. 2008, Article ID 872190, 11 pages, 2008.

[28] M. Eshaghi-Gordji, S. Kaboli-Gharetapeh, M. S. Moslehian, and S. Zolfaghari, "Stability of a mixed type additive, quadratic, cubic and quartic functional equation," in Nonlinear Analysis and Variational Problems, P. M. Pardalos, Th. M. Rassias, and A. A. Khan, Eds., Springer Optimization and Its Applications, 35, chapter 6, pp. 65-80, Springer, Berlin, Germany, 2009. 